\title{
Effects of Qigong Training on Health-Related Quality of Life, Functioning, and Cancer-Related Symptoms in Survivors of Nasopharyngeal Cancer: A Pilot Study
}

\author{
Shirley S. M. Fong, ${ }^{1}$ Shamay S. M. Ng, ${ }^{2}$ W. S. Luk, ${ }^{3}$ Louisa M. Y. Chung, \\ Janet Y. H. Wong, ${ }^{5}$ and Joanne W. Y. Chung ${ }^{4}$ \\ ${ }^{1}$ Institute of Human Performance, University of Hong Kong, Pokfulam, Hong Kong \\ ${ }^{2}$ Department of Rehabilitation Sciences, Hong Kong Polytechnic University, Hung Hom, Hong Kong \\ ${ }^{3}$ The Association of Licentiates of the Medical Council of Hong Kong, Hong Kong \\ ${ }^{4}$ Department of Health and Physical Education, Hong Kong Institute of Education, Tai Po, Hong Kong \\ ${ }^{5}$ School of Nursing, Li Ka Shing Faculty of Medicine, University of Hong Kong, Pokfulam, Hong Kong
}

Correspondence should be addressed to Shirley S. M. Fong; smfong@hku.hk

Received 30 April 2014; Accepted 14 May 2014; Published 28 May 2014

Academic Editor: Manuel Arroyo-Morales

Copyright (C) 2014 Shirley S. M. Fong et al. This is an open access article distributed under the Creative Commons Attribution License, which permits unrestricted use, distribution, and reproduction in any medium, provided the original work is properly cited.

\begin{abstract}
This study aimed to investigate the effects of Qigong intervention on quality of life (QOL), health-related functioning, and cancerrelated symptoms in survivors of nasopharyngeal cancer (NPC). Twenty-five survivors of NPC were included in the experimental group (mean age \pm SD: $55.4 \pm 7.5$ years) and 27 in the control group (mean age \pm SD: $58.7 \pm 9.5$ years). The experimental group underwent a weekly 1.5-hour Qigong training program and an identical home program (three times/week) for six months. The control group received no training. Global health status/QOL, functioning, and cancer-related symptoms were assessed by the European Organization for Research and Treatment of Cancer QLQ-C30 and QLQ-H\&N35 questionnaires before training began, after three months of Qigong training, at the end of the six-month Qigong intervention (i.e., posttest), and six months posttest. Intention-to-treat analysis revealed no statistically $(P>0.05)$ or clinically significant improvement in global health status/QOL, functioning, or symptoms in either group. The experimental group had $45.8 \%$ fewer sense-related (smell and taste) problems $(P<$ $0.05)$ but $98.6 \%$ more speech-related problems $(P<0.05)$ than the control group after the Qigong intervention. Qigong training resulted in no apparent improvement in health-related QOL, functionality, or cancer-related symptoms in cancer-free survivors of NPC, except for a possible reduction in smell- and taste-related problems.
\end{abstract}

\section{Introduction}

Many people in Southeast Asia, Southern China, and North Africa are diagnosed with nasopharyngeal cancer (NPC), a disease endemic in these regions [1]. The incidence rate is high in these areas, ranging from 25 to 50 per 100,000 persons [1]. Although advances in NPC treatments have led to improvements in survival rates (five-year survival rate $=$ $55 \%-90 \%$ ) [2], survivors often live with numerous canceror treatment-related symptoms that cause dysfunctions and a reduction in quality of life (QOL) [3, 4]. A previous study reported that survivors of NPC had a lower health-related
QOL during their cancer-free survival period than the healthy control participants. Socioeconomic status was the major determinant of QOL in this group. Participants who had a lower economic status tended to have a lower QOL [3]. It is thus necessary to identify low-cost remedial strategies to improve QOL and reduce adverse side effects in survivors of NPC.

Qigong is a low-cost mind-and-body exercise that originated in China. It comprises meditation, coordinated breathing, and graceful bodily movements [5]. There is some evidence that Qigong can improve QOL and reduce side effects of cancer treatment [6-8]. Several studies have reported 
the positive effects of Qigong training on global QOL as measured by the European Organization for Research and Treatment of Cancer (EORTC) Quality of Life Core Questionnaire 30 (QLQ-C30) [7, 8]. Cancer treatment side effects, such as constipation [7] and inflammation, were reduced $[7,8]$ and perceived cognitive abilities improved [8]. These promising results reported in cancer patients raised the possibility that survivors of NPC may also benefit from Qigong training after the conventional treatment period. To date, no study has examined the effect of Qigong intervention on residual cancer- or treatment-related side effects and QOL in NPC survivors, who tend to be young and active members of the workforce [2].

The aim of this prospective, nonrandomized, and controlled trial was to investigate the effects of Qigong training on health-related QOL, functions, and cancer-related symptoms in survivors of NPC. The research hypotheses of this study were (1) improvements in QOL and functioning and a reduction in symptoms should be observed in the experimental group across the four assessment time points (i.e., pretest, midintervention, posttest, and follow-up) and (2) the experimental group should have better QOL, higher functions, and fewer cancer-related symptoms than the control group immediately after Qigong intervention and also at the six-month follow-up point.

\section{Methods}

2.1. Participants. Survivors of NPC were recruited from a medical clinic and a cancer self-help group by convenience sampling. They were first screened by a medical doctor to ensure that inclusion and exclusion criteria were met. The inclusion criteria were as follows: (1) individuals had a history of NPC (i.e., positive Epstein-Barr virus DNA and biopsy test results) but were free from the disease during the study period; (2) had completed all cancer treatments (e.g., chemotherapy and radiotherapy) although conventional medical care was allowed; (3) were medically stable; (4) aged between 40 and 85 years; (5) had expected survival period of more than one year; (6) spoke Cantonese; (7) residing in Hong Kong; (8) had normal cognitive function; and (9) could read Chinese. The exclusion criteria were (1) receiving alternative and complementary therapies (e.g., traditional Chinese medicine); (2) having significant chronic medical conditions such as uncontrolled hypertension; (3) having significant neurological, musculoskeletal, cardiopulmonary, or vascular disorders; (4) ambulating dependently; and (5) smoking. Ethical approval was obtained from the ethics review committee of the administering institutes. Written informed consent was obtained from each participant prior to data collection. All procedures were conducted according to the Declaration of Helsinki principles.

2.2. Intervention. The intervention group received weekly 1.5-hour face-to-face Qigong training continuously for six months. The traditional 18 Forms Tai Chi Internal Qigong [5] and NPC-targeted exercises were included in the Qigong training program (Table 1). This training protocol specific for
TABLE 1: Qigong exercise protocol.

\begin{tabular}{lc}
\hline Exercise type & Duration \\
\hline Warm-up: jogging & 5 minutes \\
18 Forms Tai Chi Internal Qigong [5] & 40 minutes
\end{tabular}

(1) Raising the arms and pressing down

(2) Opening the chest

(3) Painting a rainbow from side to side

(4) Separating the clouds with two arms

(5) Rolling the arms in a horse-riding stance

(6) Rowing the boat

(7) Carrying a ball in front of the shoulders

(8) Turning around to look at the moon

(9) Twisting the waist and pushing the palms

(10) Cloud hands in a horse-riding stance

(11) Scooping the sea and searching the sky

(12) Pushing waves in walking stance

(13) Flying dove spreads its wings

(14) Punching in horse stance

(15) Flying like wild geese

(16) Rotating a flying wheel

(17) Stepping whilst bouncing a ball

(18) Pressing down to balance the chi

Additional NPC-targeted exercises

40 minutes

(1) Arms cross in front and curl up the body

(2) Place hands behind the neck

(3) Olfactory stimulation (e.g., breathe in slowly and deeply, imagine smelling a flower)

(4) Inner ear stimulation (e.g., different head and neck movements)

(5) Oral and swallowing exercise (e.g., use the tip of the tongue to massage the maxilla and swallow saliva bit by bit)

(6) Mouth-opening exercise and make sounds with the throat

Cooldown: gentle whole-body stretching 5 minutes

survivors of NPC was designed by a medical doctor and a Qigong master. It places more emphasis on the olfactory, inner ear, and oral stimulations when compared to the traditional Qigong forms. Similar to other Qigong training regimes, our training program emphasized meditation, coordinated breathing, and slow and smooth Tai Chi movements. Participants progressively learned one to two new techniques in each face-to-face session (Table 1). A certified Qigong instructor and an assistant instructor delivered all the Qigong classes. Moreover, a Qigong home program was prescribed to the intervention group to increase the training frequency to four times per week in total. The home exercises were exactly the same as those learned in the supervised sessions. The Qigong instructors checked the participants' compliance to the home program by asking and testing them every week to ensure that the training volume was adequate. All 
TABLE 2: Characteristics of participants measured at baseline.

\begin{tabular}{|c|c|c|c|}
\hline & $\begin{array}{l}\text { Qigong group } \\
\quad(n=25)\end{array}$ & $\begin{array}{l}\text { Control group } \\
\quad(n=27)\end{array}$ & $P$ \\
\hline Age (year) & $55.4 \pm 7.5$ & $58.7 \pm 9.5$ & 0.172 \\
\hline $\begin{array}{l}\text { Sex (number of } \\
\text { male : female) }\end{array}$ & $12: 13$ & $16: 11$ & 0.416 \\
\hline Weight $(\mathrm{kg})$ & $59.5 \pm 15.1$ & $56.8 \pm 10.5$ & 0.451 \\
\hline Height $(\mathrm{cm})$ & $163.2 \pm 9.1$ & $161.5 \pm 8.1$ & 0.496 \\
\hline Body mass index $\left(\mathrm{kg} / \mathrm{m}^{2}\right)$ & $22.3 \pm 5.0$ & $21.7 \pm 3.3$ & 0.640 \\
\hline \multicolumn{4}{|l|}{$\begin{array}{l}\text { Reported NPC stage at } \\
\text { diagnosis [9] }\end{array}$} \\
\hline Stage I $(n, \%)$ & $5(20 \%)$ & $2(7.4 \%)$ & \\
\hline Stage II $(n, \%)$ & $5(20 \%)$ & $7(25.9 \%)$ & \\
\hline Stage III $(n, \%)$ & $11(44 \%)$ & $15(55.6 \%)$ & \\
\hline Stage IV $(n, \%)$ & $4(16 \%)$ & $3(11.1 \%)$ & \\
\hline Post-NPC duration (year) & $12.5 \pm 7.1$ & $8.4 \pm 9.7$ & 0.094 \\
\hline \multicolumn{4}{|l|}{ Previous NPC treatment } \\
\hline Radiotherapy $(n, \%)$ & $17(68 \%)$ & $9(33.3 \%)$ & \\
\hline $\begin{array}{l}\text { Radiotherapy and } \\
\text { chemotherapy }(n, \%)\end{array}$ & $7(28 \%)$ & $18(66.6 \%)$ & \\
\hline $\begin{array}{l}\text { Radiotherapy, } \\
\text { chemotherapy, and } \\
\text { surgery }(n, \%)\end{array}$ & $1(4 \%)$ & $0(0 \%)$ & \\
\hline $\begin{array}{l}\text { Habitual physical activity } \\
\text { level (metabolic equivalent } \\
\text { hours per week) }\end{array}$ & $13.9 \pm 14.1$ & $14.9 \pm 14.5$ & 0.795 \\
\hline
\end{tabular}

Mean \pm standard deviation presented for continuous variables.

${ }^{*} P<0.05$ for between-group comparisons.

participants stopped practicing Qigong during the followup period. The control group received no Tai Chi or Qigong training but continued to receive conventional medical care if necessary throughout the whole study period.

2.3. Outcome Measurements. Demographic characteristics, habitual physical activity level (metabolic equivalent hours per week), and medical history of the participants were obtained at pretest (Table 2). In addition, all of the participants were required to complete two questionnairesEORTC QLQ-C30 and the head and neck cancer supplementary module (QLQ-H\&N35) - to evaluate their global health status/QOL, functioning and cancer-related symptoms one week before the Qigong intervention (pretest), after three months of Qigong training (midintervention test), immediately after the six-month Qigong intervention (posttest), and six months posttest (follow-up test).

The EORTC QLQ-C30 (version 3.0 for the Hong Kong Chinese population) and the QLQ-H\&N35 (Hong Kong Chinese version) were used together as originally designed [10]. These self-administered questionnaires were validated for the Hong Kong Chinese population and their psychometric properties are considered to be satisfactory [11, 12]. The QLQ-C30 questionnaire, suitable for a board range of cancer patients and survivors, comprises 30 items assessing healthrelated QOL. It is composed of one global health status/QOL scale, five functional scales (i.e., physical, role, cognitive, emotional, and social), three symptom scales (i.e., fatigue, nausea and vomiting, and pain), and six single items (i.e., dyspnea, insomnia, appetite loss, constipation, diarrhea, and financial difficulties). Items on the functional and symptom scales were scored with a four-point Likert scale $(1=$ not at all, $2=$ a little, $3=$ quite a bit, and $4=$ very much) while items in the global health status/QOL domain were scored on a sevenpoint Likert scale, ranging from 1 (very poor) to 7 (very good) [10].

The QLQ-H\&N35 module was used to assess symptoms specifically in head and neck cancer patients and survivors. It consists of 35 items in total that were scored with a fourpoint Likert scale $(1=$ not at all, $2=$ a little, $3=$ quite a bit, and 4 = very much). Seven scales assess symptoms of pain, swallowing, senses (smell/taste), speech, social eating, social contact, and sexuality, and six single items assess the presence of symptomatic problems associated with teeth, mouth opening, dry mouth (xerostomia), sticky saliva, coughing, and ill feeling [10]. Both the EORTC QLQ-C30 and QLQH\&N35 were scored according to the EORTC QLQ-C30 scoring manual [10]. In brief, all of the scale and item raw scores were linearly transformed into a 0 to 100 scale. A higher score represents a higher level of functioning on the QLQ-C30 functional scale or a higher level of global health status on the global health status/QOL scale. However, a higher score on the QLQ-C30/QLQ-H\&N35 symptom scale indicates more symptoms or problems, suggesting a greater impairment in QOL [10].

2.4. Statistical Analysis. The Kolmogorov-Smirnov test was used to check normality of all continuous data. Independent $t$-tests (for continuous variables) and chi-square tests (for categorical variables) were used to compare the demographic characteristics and all outcome variables of the Qigong and control groups before the training intervention began (baseline). Intention-to-treat analysis, specifically the last observation carried forward method, was employed to handle the missing data. To assess the time-by-group interaction effect of Qigong training on the global health status, twoway repeated measures analysis of covariance (ANCOVA) with mixed design (within-subject factor: time and betweensubject factor: group) was used.

In addition, two-way repeated measures multivariate analysis of covariance (MANCOVA) was performed to evaluate the overall time-by-group interaction, group and time effects of Qigong training on (1) functional scale outcomes and (2) symptom scale outcomes of EORTC QLQ-C30 and QLQ-H\&N35. Multivariate tests were used instead of univariate tests to avoid an increase in type I error associated with multiple comparisons. If there were significant betweengroup differences in any outcome variables at baseline, these outstanding outcomes were treated as covariates in the multivariate and univariate tests mentioned above. Effect sizes (partial eta-squared) were also reported. By convention, values of $0.14,0.06$, and 0.01 represent large, medium, and small effects, respectively [13]. 
If the two-way repeated measures MANCOVA or ANCOVA revealed significant effects overall, follow-up analyses were performed using one-way repeated measures analysis of variance (ANOVA) and post hoc paired $t$-tests with Bonferroni correction as appropriate. Independent $t$-tests were also used to compare the outstanding outcome variables between the two groups. The significance level (alpha) was set at 0.05 (two-tailed) for all the tests except the paired $t$-tests (alpha was set at 0.008 due to six pairwise comparisons). All statistical analyses were performed using the IBM Statistical Package for the Social Sciences (SPSS) version 20.0 software (IBM, Armonk, NY, USA).

\section{Results}

Fifty-two survivors of NPC were eligible to join the study and completed the baseline assessments. Twenty-five participants underwent Qigong training voluntarily for six months while twenty-seven participants acted as controls. At the end of the study, 14 participants in the experimental group had completed the Qigong training and the follow-up tests (i.e., dropout rate $=44.0 \%$ ) and 21 participants in the control group had completed the follow-up tests (i.e., dropout rate $=22.2 \%$ ). The reasons for dropping out were similar between the two groups. For the Qigong group, subjects who left the trial reported they were busy $(n=4)$, sick $(n=2)$, or unable to commit to the time $(n=5)$. Those control group subjects who stopped participating were busy $(n=1)$, unable to commit to the time $(n=1)$, overseas $(n=1)$, or unreachable $(n=3)$. No adverse effects were reported during the in-class Qigong training or the home practice. The Qigong class attendance rate was on average approximately $90 \%$.

3.1. Comparison of Baseline Data. No significant differences were found in any of the demographic (Table 2) and outcome variables (Table 3 ) between the two groups at baseline $(P>$ 0.05 ). Therefore, analysis of variance (ANOVA) instead of ANCOVA was performed in all the univariate and multivariate tests.

3.2. Global Health Status/QOL. Two-way repeated measures ANOVA results revealed that the group $\times$ time interaction and group effects were not significant $(P>0.05)$. However, the time effect was significant $(P=0.011)$ (Table 3$)$. Post hoc paired $t$-tests showed that the global health status score, representing health-related QOL, was $12.6 \%$ higher at posttest $(P=0.001)$ and $15.9 \%$ higher at followup $(P=0.006)$ when compared to the pretest value of the control group. No significant differences $(P>0.05)$ were found in other pairwise comparisons between time points (Table 3 ).

3.3. EORTC QLQ-C30 Functional Scale Outcomes. The $P$ values and effect sizes of the multivariate analysis are presented in Table 3 . No significant group $\times$ time, group or time effects $(P>0.05)$ were found. Therefore follow-up univariate analyses were not performed.

3.4. EORTC QLQ-C30 Symptom Scale Outcomes. Similar to the QLQ-C30 functional scale outcomes, no significant group $\times$ time, group or time effects $(P>0.05)$ were found in the multivariate analysis (Table 3 ).

3.5. EORTC QLQ-HぬN35 Symptom Scale Outcomes. MANOVA results showed that the group $\times$ time interaction and time effects were not significant $(P>0.05)$. Only the group effect was significant $(P=0.003)$. Follow-up independent $t$-tests identified significant between-group differences at $(1)$ midintervention $(P=0.027)$, posttest $(P=0.017)$, and follow-up test $(P=0.006)$ for the sense problems outcome and $(2)$ midintervention $(P=0.038)$ and posttest $(P=0.034)$ for the speech problems outcome (Table 3). The control group had higher scores for senserelated (smell and taste) problems but lower speech-related problem scores than the Qigong group during and after the Qigong intervention (Table 3).

\section{Discussion}

This is the first study to assess the impact of Qigong training on QOL for cancer-free survivors of NPC. Our results show that Qigong training did not improve healthrelated QOL for the intervention group. Instead global health status/QOL improved in the no-training control group. Our findings are in contrast to previous studies and do not support our hypotheses. Qigong has been proposed to improve QOL in cancer patients $[7,8,14]$ through several possible mechanisms, including reducing the side effects of conventional cancer treatments and cancer-related symptoms [7], decreasing inflammation [7, 8], and increasing cognitive function [8]. A major reason for the discrepancy in findings between our study and the previous studies could be the difference in the participants' characteristics. Previous randomized controlled trials included heterogeneous cancer patients (e.g., breast cancer, lymphoma, and lung cancer) who had received/were receiving different forms of conventional cancer treatments. All of them were recruited from hospitals $[7,8]$. Newly diagnosed cancer patients and patients in the treatment stage could be more prone to anxiety, depression, and other psychological impairments than rehabilitated survivors of cancer [15]. Hence, they may benefit more from Qigong training in terms of improving QOL $[7,8]$.

Our study included survivors of NPC exclusively. They were recruited from a medical clinic and a cancer self-help group. All of them were cancer-free during the study period. The post-NPC duration for the Qigong group was 12.5 years while for the control group it was 8.4 years. They may thus have fewer symptoms and functional impairments and so better health-related QOL overall when compared to hospitalized cancer patients. The mean global health status/QOL score of the Qigong group (61.8) actually exceeded the EORTC QLQ-C30 reference value (61.3) at pretest [16]. Thus, we postulate that Qigong training may not improve their relatively high level of QOL further. Instead, spontaneous improvement could heighten their health-related QOL, as demonstrated in the no-training control group. Further study should include a larger sample and adopt a randomized controlled study design to investigate this hypothesis. In addition, qualitative studies (e.g., structured interviews) could 


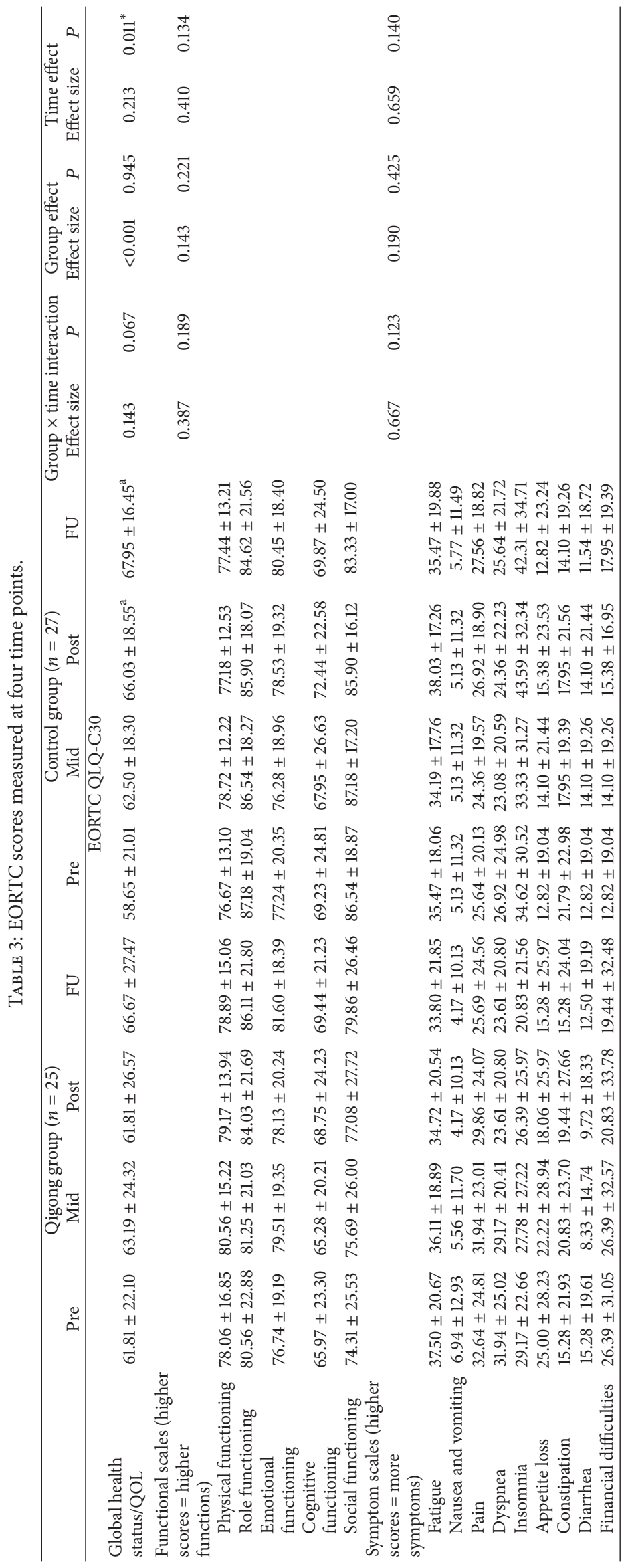




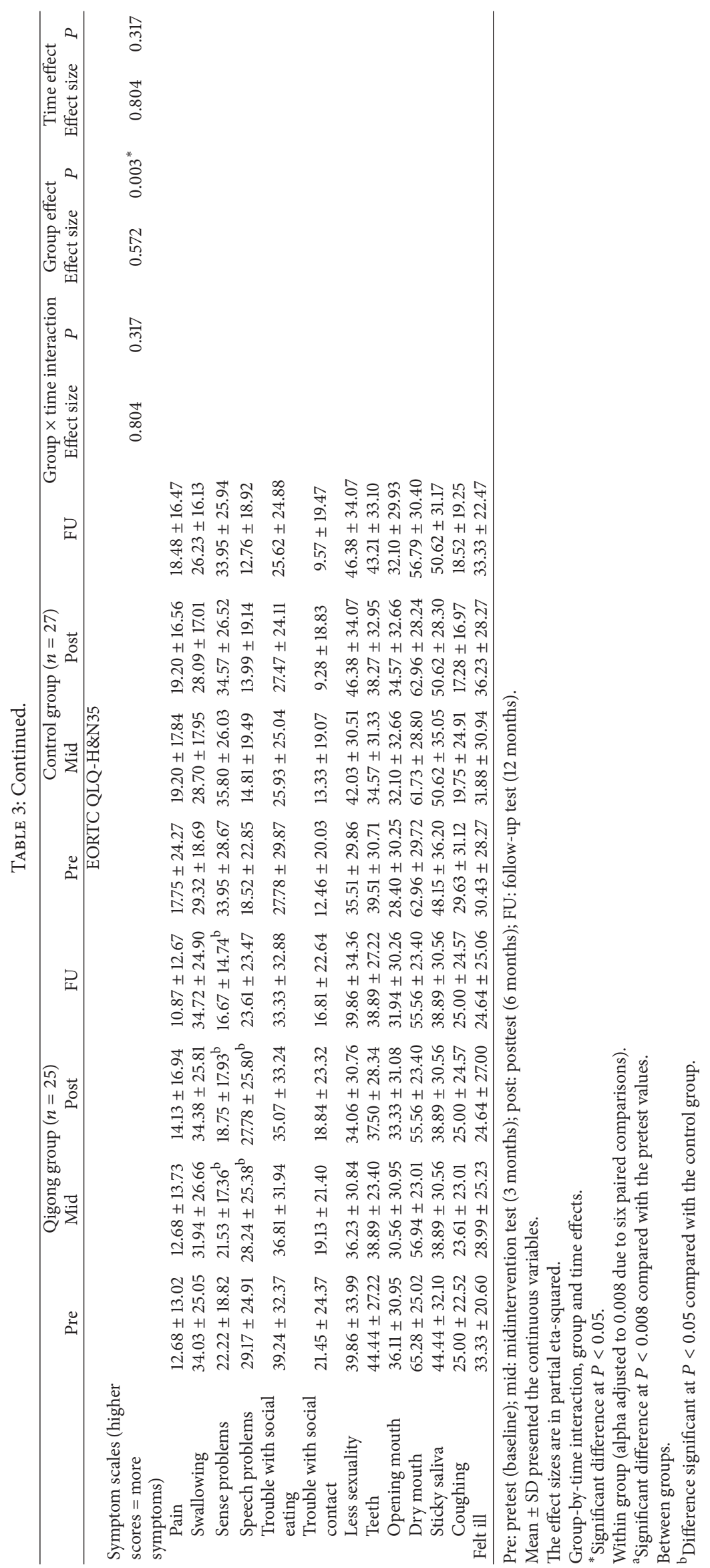


be carried out to discover the reasons for the spontaneous improvement in QOL among survivors of NPC.

The results also revealed that, over the study period, neither the Qigong group nor the control group showed significant improvements in physical, role, emotional, cognitive, or social functions. These findings are not entirely surprising given the fact that our participants had generally higher EORTC QLQ-C30 functional scores than the norm [16] at baseline. Perhaps no further improvements in functioning were possible regardless of the stimulus. This is the first study to include a group of rehabilitated survivors of NPC. Comparing our results with those obtained for cancer patients $[7,8]$ may not be appropriate. Further randomized controlled trials should be carried out to explore whether or not Qigong training can further improve functions in survivors of NPC.

Among all of the general cancer symptoms (QLQ-C30 symptom scores) and NPC-specific symptoms (QLQ-H\&N35 symptom scores) measured, only the sense-related problems score was lower in the Qigong group than in the control group during and after the intervention period. The Qigong group continued to have fewer sense-related (smell and taste) problems than the control group up to six months after the cessation of training. Our findings are in line with a previous case report that suggested that smelling and taste in the mouth changed during Qigong practice [17]. These findings are encouraging because chronic smell and taste disorders are common among survivors of NPC $[18,19]$. These consequences of NPC and/or side effects of chemotherapy and radiotherapy can impair food intake and so may lead to nutritional deficits and diminished QOL $[18,20,21]$. However, no effective curative strategy has been developed [18, 22, 23].

Qigong has potential use as a remedial intervention to improve perceived taste and smell in survivors of NPC. During the NPC-targeted exercises of the Qigong intervention, participants were requested to massage their oral cavity with their tongue (Table 1). We postulated that this technique might stimulate the taste buds, cortical representation of taste [24], and saliva production, which are essential for the taste sensation [18]. In addition, the olfactory stimulation exercises required the participants to breathe in slowly and deeply as if they were smelling a flower (Table 1). This kind of exercise might stimulate the olfactory receptors located in the nasal cavity, which are crucial for smell and taste sensations [18]. Further study should evaluate smell and taste sensations objectively, for example, using the "Sniffin' Sticks" test [19], to exclude possible psychological or placebo effects before suggesting Qigong training for NPC survivors.

Another statistically significant finding is that the Qigong group experienced more speech problems than the control group at posttest and follow-up test. This finding was unanticipated as a previous study found that Qigong could improve speech fluency in people who stutter, as they gained more control over muscular tension after the exercise [25]. The speech-related problems considered in the QLQ-H\&N35 questionnaire concerned hoarseness and trouble talking to others [10], which may not be directly related to muscular control of sound production. Further study should explore whether the increased speech problems observed are due to Qigong training, aging, smell and taste problems, and so forth.

The Qigong training intervention was not able to alleviate symptoms such as fatigue, pain, insomnia, and swallowing problems in survivors of NPC (Table 3 ). This may be because our participants had been fully rehabilitated and did not have residual cancer-related problems [16]. As a result, Qigong training might not be able to further lessen their symptoms.

This study has several limitations and findings should be interpreted with caution. First, the group assignment was non-randomized. Participants in the Qigong group may have been more motivated to seek treatments or training, and, therefore, they may have had fewer symptoms (e.g., sense-related problems) than the control group [13]. Second, due to the nature of the Qigong intervention, blinding of participants was not possible. In addition, we used self-report questionnaires to measure outcomes, so bias/placebo effects (e.g., participants perceive they feel better after Qigong training) cannot be excluded. Third, the sample size is rather small that may have compromised the statistical power leading to the insignificant results [26]. Finally, all of the participants were cancer-free survivors. The results cannot be generalized to patients of NPC who may have more symptoms and poorer functions. The effect of Qigong training on patients with NPC requires further investigation. Moreover, further study should take confounding factors (e.g., social support) into account when measuring QOL in survivors of NPC.

\section{Conclusions}

In summary, this clinical trial indicated that Qigong training confers no apparent improvement on health-related quality of life, functioning, or cancer-related symptoms in survivors of NPC. Further study is required to determine whether the improvement in taste- and smell-related problems perceived by survivors of NPC during and after Qigong training was due to actual recovery of senses or due to the placebo effect only.

\section{Conflict of Interests}

The authors declare that they have no conflict of interests with respect to the authorship or publication of this paper.

\section{Acknowledgments}

This study was supported by a Seed Fund for Basic Research for New Staff (201308159012) from the University of Hong Kong and an Internal Research Grant (RG57/2012-2013R) from the Hong Kong Institute of Education. The authors would like to thank Professor L. Lam for providing advice on the use of questionnaires and Professor Rich Masters for his intellectual input, advice, and support.

\section{References}

[1] M. Al-Sarraf and M. S. Reddy, "Nasopharyngeal carcinoma," Current Treatment Options in Oncology, vol. 3, no. 1, pp. 21-32, 2002. 
[2] M. Agulnik and J. B. Epstein, "Nasopharyngeal carcinoma: current management, future directions and dental implications," Oral Oncology, vol. 44, no. 7, pp. 617-627, 2008.

[3] F.-M. Fang, H.-C. Chiu, W.-R. Kuo et al., "Health-related quality of life for nasopharyngeal carcinoma patients with cancerfree survival after treatment," International Journal of Radiation Oncology, Biology, Physics, vol. 53, no. 4, pp. 959-968, 2002.

[4] M. Cengiz, E. Ozyar, M. Esassolak et al., "Assessment of quality of life of nasopharyngeal carcinoma patients with EORTC QLQ-C30 and H\&N-35 modules," International Journal of Radiation Oncology, Biology, Physics, vol. 63, no. 5, pp. 13471353, 2005.

[5] Y. K. Mak, 18 Forms Tai Chi Qigong, Wan Li Book Co, Hong Kong, China, 7th edition, (Chinese).

[6] S. S. M. Fong, S. S. M. Ng, W. S. Luk et al., "Shoulder mobility, muscular strength, and quality of life in breast cancer survivors with and without Tai Chi Qigong training," Evidence-Based Complementary and Alternative Medicine, vol. 2013, Article ID 787169, 6 pages, 2013.

[7] B. Oh, P. Butow, B. Mullan, and S. Clarke, "Medical Qigong for cancer patients: pilot study of impact on quality of life, side effects of treatment and inflammation," The American Journal of Chinese Medicine, vol. 36, no. 3, pp. 459-472, 2008.

[8] B. Oh, P. N. Butow, B. A. Mullan et al., "Effect of medical Qigong on cognitive function, quality of life, and a biomarker of inflammation in cancer patients: a randomized controlled trial," Supportive Care in Cancer, vol. 20, no. 6, pp. 1235-1242, 2012.

[9] American Joint Committee on Cancer, AJCC Cancer Staging Manual, Lippincott Williams \& Wilkins, Philadelphia, Pa, USA, 5th edition, 1997.

[10] P. M. Fayers, N. K. Aaronson, K. Bjordal, M. Groenvold, D. Curran, and A. Bottomley, The EORTC QLQ-C30 Scoring Manual, European Organisation for Research and Treatment of Cancer, Brussels, Belgium, 3rd edition, 2001.

[11] H. Zhao and K. Kanda, "Translation and validation of the standard Chinese version of the EORTC QLQ-C30," Quality of Life Research, vol. 9, no. 2, pp. 129-137, 2000.

[12] H. Zhao and K. Kanda, "Testing psychometric properties of the standard Chinese version of the European Organization for Research and Treatment of Cancer Quality of Life Core Questionnaire 30 (EORTC QLQ-C30)," Journal of Epidemiology, vol. 14, no. 6, pp. 193-203, 2004.

[13] L. G. Portney and M. P. Watkins, Foundations of Clinical Research: Applications to Practice, Pearson Education, Upper Saddle River, NJ, USA, 3rd edition, 2009.

[14] Y. Zeng, T. Luo, H. Xie, M. Huang, and A. S. K. Cheng, "Health benefits of Qigong or Tai Chi for cancer patients: a systematic review and meta-analyses," Complementary Therapies in Medicine, vol. 22, no. 1, pp. 173-186, 2014.

[15] A. Paul, R. Lauche, H. Cramer, N. Altner, J. Langhorst, and G. J. Dobos, "An oncology mind-body medicine day care clinic: concept and case presentation," Integrative Cancer Therapies, vol. 12, no. 6, pp. 503-507, 2013.

[16] N. W. Scott, P. M. Fayers, N. K. Aaronson et al., EORTC QLQC30 Reference Values, European Organisation for Research and Treatment of Cancer, Brussels, Belgium, 2008.

[17] J. Jouper and M. Johansson, "Qigong and mindfulness-based mood recovery: exercise experiences from a single case," Journal of Bodywork and Movement Therapies, vol. 17, no. 1, pp. 69-76, 2013.
[18] P. Ravasco, "Aspects of taste and compliance in patients with cancer," European Journal of Oncology Nursing, vol. 9, supplement 2, pp. S84-S91, 2005.

[19] W.-K. Ho, D. L. W. Kwong, W. I. Wei, and J. S. T. Sham, "Change in olfaction after radiotherapy for nasopharyngeal cancer-a prospective study," American Journal of Otolaryngology: Head and Neck Medicine and Surgery, vol. 23, no. 4, pp. 209-214, 2002.

[20] J. B. Epstein and A. Barasch, "Taste disorders in cancer patients: pathogenesis, and approach to assessment and management," Oral Oncology, vol. 46, no. 2, pp. 77-81, 2010.

[21] T. B. Comeau, J. B. Epstein, and C. Migas, "Taste and smell dysfunction in patients receiving chemotherapy: a review of current knowledge," Supportive Care in Cancer, vol. 9, no. 8, pp. 575-580, 2001.

[22] S. S. Schiffman and C. A. Gatlin, "Clinical physiology of taste and smell," Annual Review of Nutrition, vol. 13, pp. 405-436, 1993.

[23] M. Rehwaldt, R. Wickham, S. Purl et al., "Self-care strategies to cope with taste changes after chemotherapy," Oncology Nursing Forum, vol. 36, no. 2, pp. E47-E56, 2009.

[24] W. S. Bornstein, "Cortical representation of taste in man and monkey II. The localization of the cortical taste area in man and a method of measuring impairment of taste in man," Yale Journal of Biology and Medicine, vol. 13, no. 1, pp. 133-156, 1940.

[25] P. Potrola and J. Goral-Potrola, "In search of new methods. Qigong in stuttering therapy," Studia Medyczne, vol. 29, no. 3, pp. 242-250, 2013.

[26] M. T. King, M. L. Bell, D. Costa, P. Butow, and B. Oh, "The Quality of Life Questionnaire Core 30 (QLQ-C30) and Functional Assessment of Cancer-General (FACT-G) differ in responsiveness, relative efficiency, and therefore required sample size," Journal of Clinical Epidemiology, vol. 67, no. 1, pp. 100-107, 2014. 


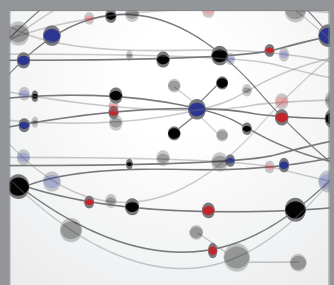

The Scientific World Journal
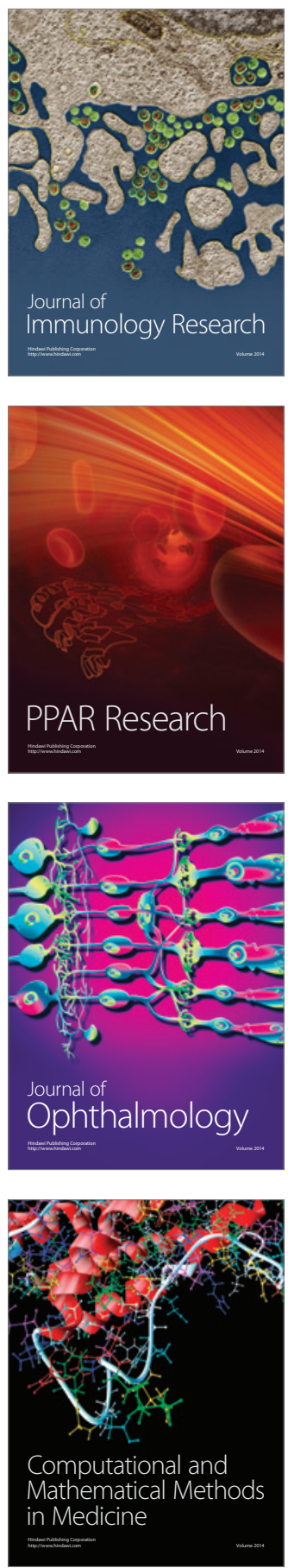

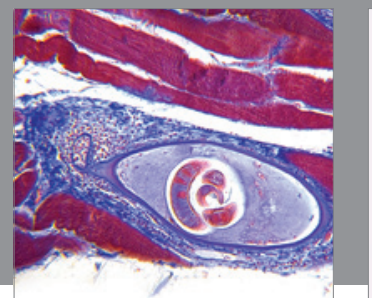

Gastroenterology

Research and Practice
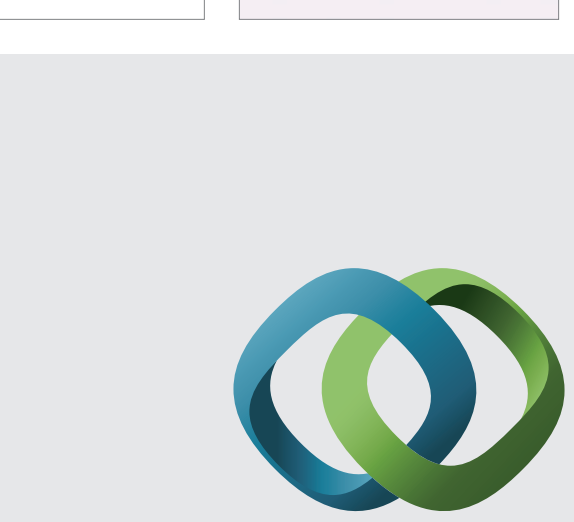

\section{Hindawi}

Submit your manuscripts at

http://www.hindawi.com
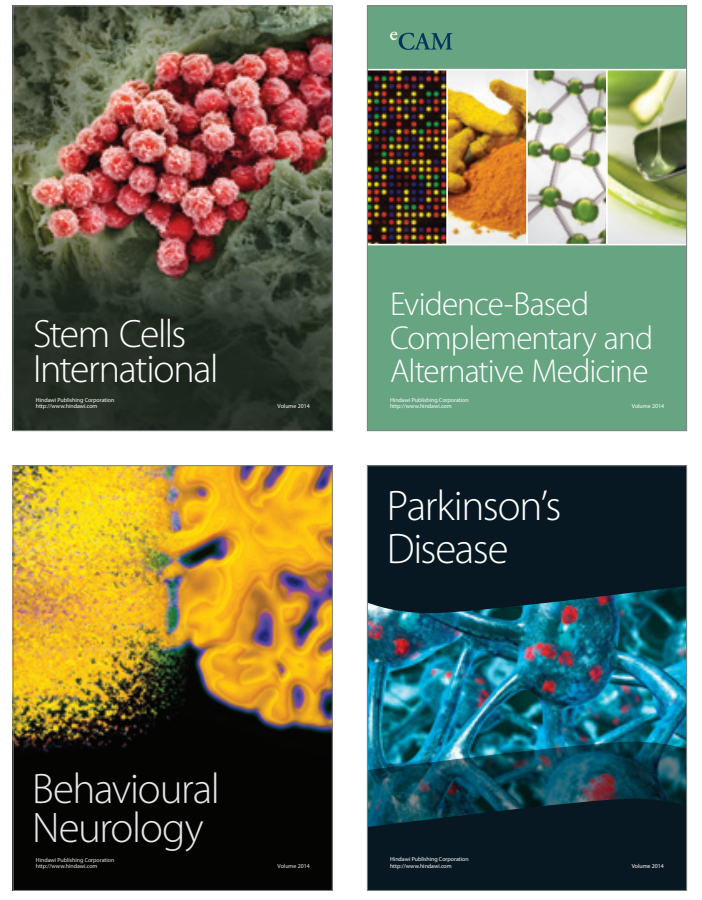
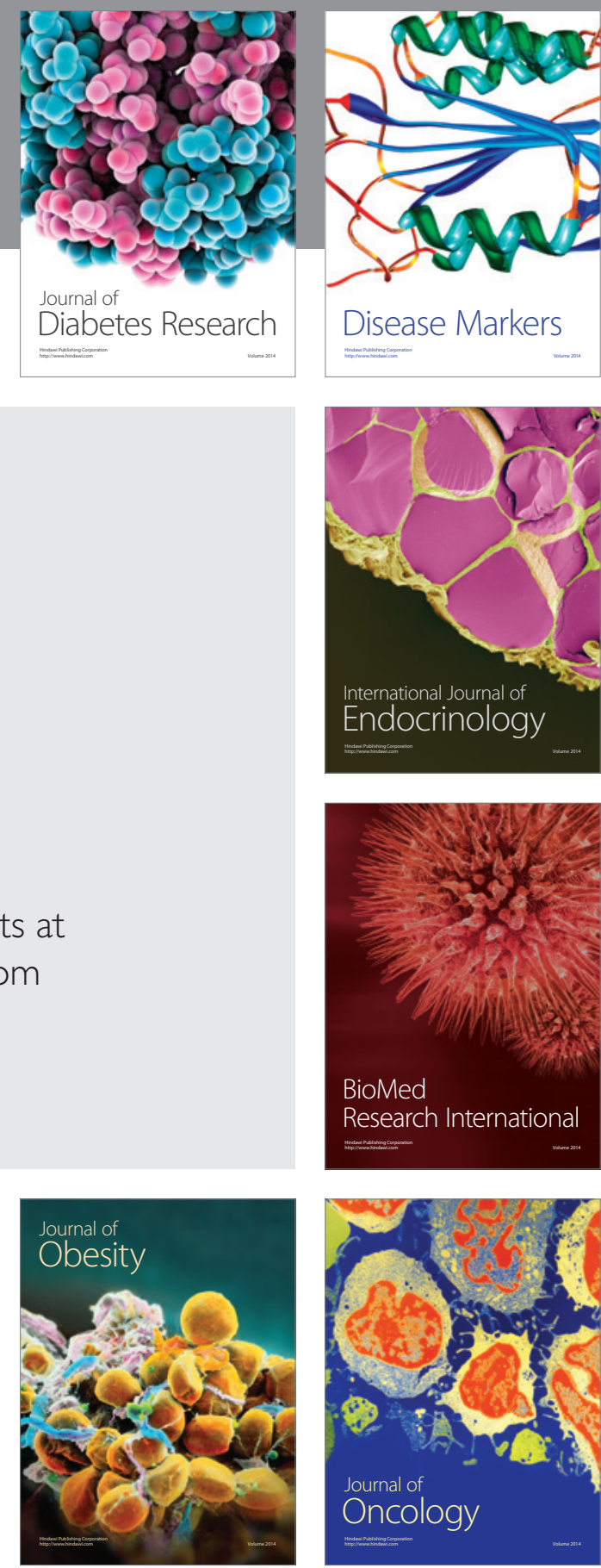

Disease Markers
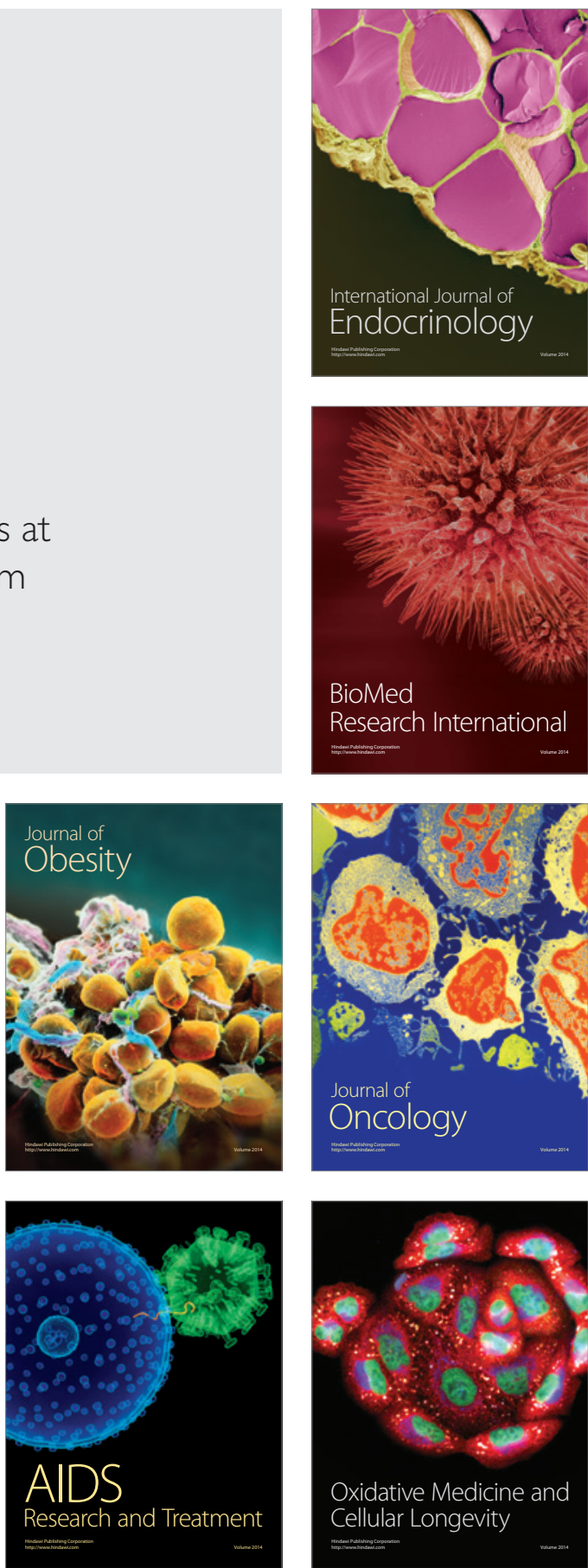